\title{
Rapid Evolution of the Photosystem II Electronic Structure during Water Splitting
}

\author{
Katherine M. Davis, ${ }^{1, \dagger}$ Brendan T. Sullivan, ${ }^{1 \dagger}$ Mark C. Palenik, ${ }^{1,}$ Lifen Yan, ${ }^{1}$ Vatsal Purohit, ${ }^{1, \Uparrow}$ Gregory Robison, ${ }^{1, \|}$ \\ Irina Kosheleva, ${ }^{2}$ Robert W. Henning, ${ }^{2}$ Gerald T. Seidler, ${ }^{3}$ and Yulia Pushkar ${ }^{1, *}$ \\ ${ }^{1}$ Department of Physics and Astronomy, Purdue University, West Lafayette, Indiana 47907, USA \\ ${ }^{2}$ Center for Advanced Radiation Sources, The University of Chicago, Chicago, Illinois 60637, USA \\ ${ }^{3}$ Department of Physics, University of Washington, Seattle, Washington 98195, USA
}

(Received 10 May 2018; revised manuscript received 18 July 2018; published 23 October 2018)

\begin{abstract}
Photosynthetic water oxidation is a fundamental process that sustains the biosphere. $\mathrm{A} \mathrm{Mn}_{4} \mathrm{Ca}$ cluster embedded in the photosystem II protein environment is responsible for the production of atmospheric oxygen. Here, time-resolved x-ray emission spectroscopy (XES) is used to observe the process of oxygen formation in real time. These experiments reveal that the oxygen evolution step, initiated by three sequential laser flashes, is accompanied by rapid (within $50 \mu \mathrm{s}$ ) changes to the Mn $K \beta$ XES spectrum. However, no oxidation of the $\mathrm{Mn}_{4} \mathrm{Ca}$ core above the all- $\mathrm{Mn}^{\mathrm{IV}}$ state is detected to precede $\mathrm{O}-\mathrm{O}$ bond formation, and the observed changes are therefore assigned to $\mathrm{O}-\mathrm{O}$ bond-formation dynamics. We propose that $\mathrm{O}-\mathrm{O}$ bond formation occurs prior to the transfer of the final (fourth) electron from the $\mathrm{Mn}_{4} \mathrm{Ca}$ cluster to the oxidized tyrosine $\mathrm{Tyr}_{Z}$ residue. This model resolves the kinetic limitations associated with $\mathrm{O}-\mathrm{O}$ bond formation and suggests an evolutionary adaptation to avoid releasing harmful peroxide species.
\end{abstract}

DOI: 10.1103/PhysRevX.8.041014

Enzymes function as nature's catalysts, facilitating virtually all the reactions necessary for life. By carefully coordinating electron dynamics and atomic rearrangements within a predefined energy landscape, they enable a broad range of efficient and highly selective transformations, many of which have proven challenging for chemists. Among these, the reaction catalyzed by the $\mathrm{Mn}_{4} \mathrm{Ca}$ cluster of photosystem II (PS II) during photosynthesis holds a special place, as the ability to biosynthesize $\mathrm{O}_{2}$ from $\mathrm{H}_{2} \mathrm{O}$ occurred only once during evolution. The development of this process dramatically altered our planet by generating the oxygen-rich atmosphere we live in today. The catalytic activity and

\footnotetext{
* Corresponding author. ypushkar@purdue.edu

${ }^{\dagger}$ Present address: Department of Chemistry, Princeton University, Princeton, NJ 08544, USA.

${ }^{\star}$ Present address: Neutron Scattering Division, Oak Ridge National Laboratory, Oak Ridge, TN 37830, USA.

${ }^{\S}$ Present address: Naval Research Laboratory, Washington, DC 20375, USA.

"Present address: Department of Biology, Purdue University, West Lafayette, IN 47907, USA.

"Present address: Department of Physics and Astronomy, Hanover College, Hanover, IN 47243, USA.

Published by the American Physical Society under the terms of the Creative Commons Attribution 4.0 International license. Further distribution of this work must maintain attribution to the author(s) and the published article's title, journal citation, and DOI.
}

Subject Areas: Biological Physics

quantum efficiency of the oxygen evolving complex (OEC) remain unmatched by synthetic systems developed for artificial photosynthesis. Despite its far-reaching consequences, the underlying mechanism of PS II remains debated.

In 1970, Kok, Forbush, and McGloin described a potential water-splitting mechanism in which the OEC cycles through five states $\left(S_{0}-S_{4}\right)$, corresponding to the oxidation states of manganese, following sequential visible-light absorptions [Fig. 1(a)] [1]. Antenna pigments from the surrounding protein matrix absorb these photons and funnel energy towards $P_{680}$, the chlorophyll $a$ special pair responsible for charge separation. Within nanoseconds, the tyrosine residue $\left(\mathrm{Tyr}_{Z}\right)$ located between $P_{680}$ and the OEC is oxidized by the special pair to form $\mathrm{Tyr}_{Z}{ }^{*}$, which is subsequently reduced by the OEC on a microsecond timescale. This process drives the water-splitting reaction [2].

The past 40 years have yielded new insights into the structure of PS II [6-15], as well as the nature and timing of the $S$-state transitions that form the Kok cycle [Fig. 1(a)] [3,16-19]. Nonetheless, the critical step during which the $\mathrm{O}-\mathrm{O}$ bond is formed remains poorly characterized and, thus, cannot be implemented in artificial systems. $\mathrm{O}-\mathrm{O}$ bond formation likely occurs on a microsecond timescale during the $S_{3}$-to- $S_{0}$ transient step of the catalytic cycle, culminating in $\mathrm{O}_{2}$ evolution. Direct monitoring of this transient process, however, has proven challenging, and details remain elusive. A preeminent report by Babcock, Blankenship, and Sauer, as well as recent studies by Nilsson et al., support a rate of $\mathrm{Tyr}_{Z}{ }^{*}$ reduction with $t_{1 / 2} \sim 1 \mathrm{~ms}$ following three flashes and associates this rate 
(a)
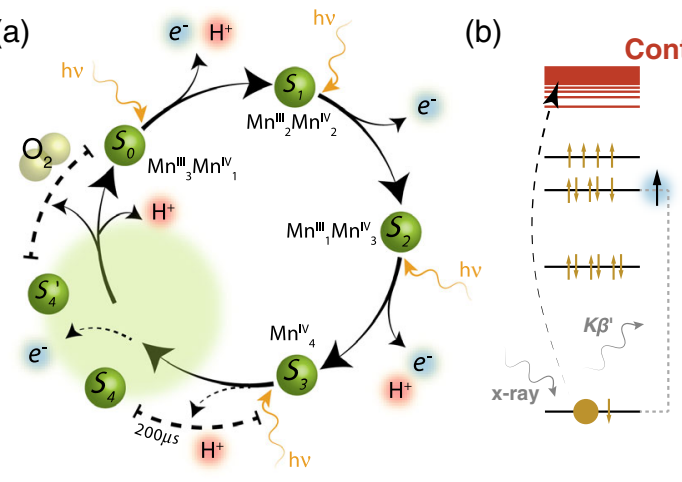

(1)

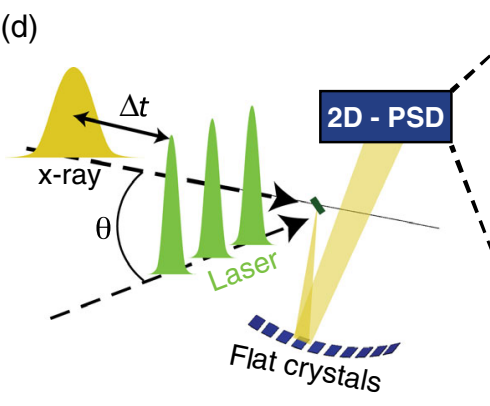

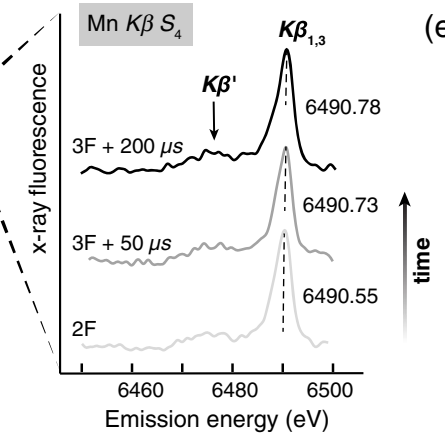
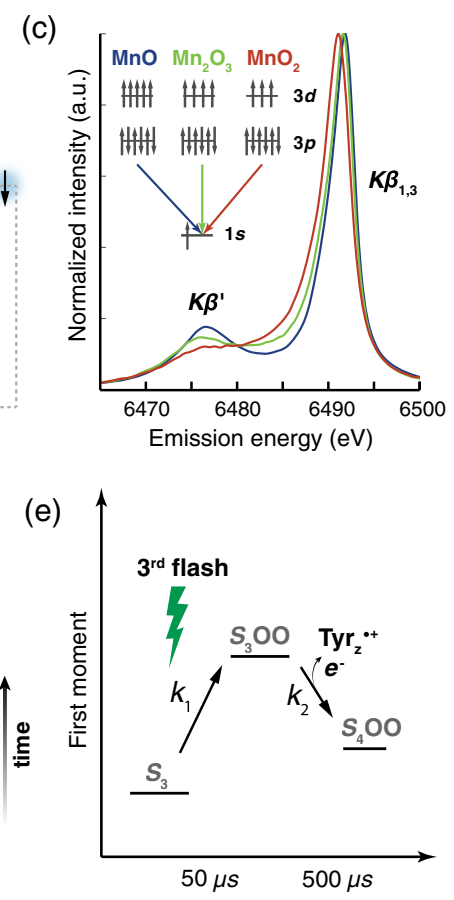

FIG. 1. (a) Current model of the Kok cycle. Depiction of sequential incident visible-light photon absorptions triggering electron or proton release [3]. The dashed region is based on previous analysis of the $S_{3}$-to- $S_{0}$ transition in which the $S_{4}$ state is proposed [3,4]. (b) Electronic transitions, reflected in the $K \beta$ main lines, are influenced by the spin state of the Mn ion. (c) A spectral comparison of Mn oxides depicts the effect of oxidation state on the $K \beta$ emission lines. (d) Nanosecond laser pulses (1, 2, or 3) are used to advance the Kok cycle in the protein (Table S1 [5]). The pump-probe delay time $\Delta t$, measured from the final laser flash to the center of the $\mathrm{x}$-ray pulse, is set dependent on the desired $S$ state. X-ray fluorescence from the sample is reflected by ten flat analyzer crystals onto a $2 \mathrm{D}$-positionsensitive detector. $K \beta$ emission spectra are extracted to form snapshots of the electronic structure in time. Smoothed emission spectra are presented for $2 F$ (majority $S_{3}$ ) and two time points during the $S_{3}$-to- $S_{0}$ transition. (e) The proposed reaction scheme shows the early evolution of the OEC during the $S_{3}$-to- $S_{0}$ transition, providing an interpretation of spectroscopic results.

constant with the formation of the $S_{4}$ state, subsequently capable of fast $\mathrm{O}_{2}$ evolution [20,21]. It was pointed out later that such hypotheses face a serious kinetic challenge in that the timescale of molecular oxygen release, following the formation of the $S_{4}$ state, is very short for the associated redox chemistry and bond-formation dynamics [22]. As an alternative hypothesis, $S_{3}$-state peroxide formation was proposed [22], but this proposal has not yet been confirmed experimentally.

Given the lack of definitive spectroscopic results, computational simulations have been performed to model the $\mathrm{O}-\mathrm{O}$ bond-formation path [23-26]. Many of these imply oxidation of the OEC past $\mathrm{Mn}^{\mathrm{IV}}{ }_{4}$ via the formation of a $\mathrm{Mn}^{\mathrm{V}} \mathrm{Mn}^{\mathrm{IV}}{ }_{3}$ state, also presented as $\mathrm{Mn}^{\mathrm{IV}}{ }_{4}-\mathrm{O}^{\bullet}$ (oxyl radical). This oxidized configuration is currently associated with $S_{4}$ and would precede $\mathrm{O}-\mathrm{O}$ bond formation $[23,27]$. Experimental proof for a $\mathrm{Mn}^{\mathrm{V}} \mathrm{Mn}^{\mathrm{IV}}{ }_{3}$ intermediate state is currently lacking, and our data rule out its formation. Here, we examine the earliest dynamic in the $S_{3}$-to- $S_{0}$ transition via time-resolved $\mathrm{x}$-ray emission spectroscopy (TR XES), utilizing dispersive detection, to aid our understanding of this critical biological process [28,29]. In 2015, we proposed a new mechanistic model [Fig. 1(e)] in which $\mathrm{O}-\mathrm{O}$ bond formation occurs prior to the transfer of the final (fourth) electron from the $\mathrm{Mn}_{4} \mathrm{Ca}$ cluster to explain our preliminary spectroscopic results [30]. An x-ray crystallographic study of the $S_{3}$ state [13] recently confirmed our DFT-based proposal, producing a virtually indistinguishable $S_{3}$-state model, within the experimental resolution of x-ray diffraction [31].

Here, we deliver an extensive statistical analysis of these initial data sets in conjunction with those collected subsequently, each consistently composed of almost a half million repetitive interrogations of the OEC electronic structure. The power of large statistical data sets has long been realized, often enabling the development of new research tools. A single, blurry electron microscopy image of a complex biomolecule, for instance, provides little insight into its structure, while several thousand images can now deliver atomically resolved models [32]. In a similar fashion, repetitive measurement of a spectroscopic response allows us to solidify the rapid electronic structure evolution in the $S_{3}$-to- $S_{0}$ transition, a result which is 
TABLE I. Experimental characteristics of the pulsed $\mathrm{x}$-ray source.

\begin{tabular}{ll}
\hline \hline Characteristics & \multicolumn{1}{c}{ BioCARS } \\
\hline Excitation energy & $\begin{array}{c}\text { Peak energy } 7.85 \mathrm{keV}, \text { FWHM } \\
\text { about } 500 \mathrm{eV} \\
\end{array}$ \\
$\begin{array}{l}\text { Approximately } 45 \times 100 \mu \mathrm{m}^{2} \\
\text { Pulse length }\end{array}$ & $44 \mu \mathrm{s}(22 \mu$ s data set 5$)$ \\
Photon flux & $3 \times 10^{11}$ photons $/$ pulse \\
Dose delivered & Approximately $7 \times 10^{7}$ photons $/ \mu \mathrm{m}^{2}$ \\
per pulse & \\
\hline \hline
\end{tabular}

required to complete the description of $\mathrm{O}-\mathrm{O}$ bond formation in natural photosynthesis.

Mn $K \beta$ spectral emission lines reflect the number of unpaired $3 d$ electrons and, thus, provide information about the oxidation and/or spin states for a given $\mathrm{Mn}$ ion, inaccessible via structural methods such as $\mathrm{x}$-ray crystallography [33]. The exchange interaction between the $3 p$ hole and $3 d$ valence electrons in the final state causes multiplet splitting that results in separate $K \beta_{1,3}$ and $K \beta^{\prime}$ peaks [Figs. 1(b) and 1(c)]. This coupling is directly linked to the electronic state of Mn such that an increase in the oxidation state results in decreased splitting between the $K \beta$ spectral lines. This effect is most apparent in the $K \beta_{1,3}$ peak position shift to lower energies with increasing oxidation. XES also allows for dispersive detection, in which the full emission spectrum is recorded during a single, intense, polychromatic $\mathrm{x}$-ray pulse [Fig. 1(d)] [28]. The temporal resolution of such a setup is limited in practice only by the time structure of the x-ray source. In our experiments, we utilize multibunch $\mathrm{x}$-ray exposures of 22-44 $\mu$ s duration to match the microsecond kinetics of the OEC [3] [Table I and Fig. S1(a) [5]]. We previously determined that exposures of up to $66 \mu \mathrm{s}$ under these conditions are undamaging to PS II [28,34]. Data collection is performed using a von Hamos-style miniature x-ray emission spectrometer (miniXES) [Fig. S1(b) [5] ] [28,35], and a nonjet open-air sample delivery system [see Supplemental Material and Fig. S1(c) [5]] is used to supply fresh, unrecycled PS II for each measurement at a controlled repetition rate. Samples are excited given a defined number (0-3) of laser flashes $(F)$ and probed at a time $(\Delta t)$ after the final laser flash by a single $\mathrm{x}$-ray pulse [Figs. 1(d) and S1 (a) and Table S1 [5] ]. For clarity, we note that $0 F$, i.e. no laser flashes, corresponds to majority state $S_{1}, 1 F$ corresponds to majority state $S_{2}$, etc.

Our total data set is composed of five separate beam times, ultimately accumulating over two million x-ray pulses to measure different $S$ states (Table II). Note that data set five is analyzed separately as its background differs from the other four beamtimes due to beamline upgrades. Time-resolved spectra of the majority $S$ states $S_{1}, S_{2}$, and $S_{3}$ are collected following zero, one, and two laser flashes, respectively, with a spacing between consecutive laser flashes of $100 \mathrm{~ms}$, corresponding to a laser frequency of $10 \mathrm{~Hz}$. Samples are probed with the x-ray beam following a $\Delta t=500 \mu$ s time delay from the final laser flash to allow for the full reduction of $\operatorname{Tyr}_{Z}{ }^{\cdot}$ with limited decay of the formed $S$ state (Table S1 [5]) $[3,36]$. Given the multiplet character of the spectra and the noise inherent for such a dilute biological sample, previous studies recommend the use of the statistical first moment $\left(\sum_{j} E_{j} \cdot I_{j}\right) /\left(\sum_{j} I_{j}\right)$, surrounding the $K \beta_{1,3}$ peak, as an indicator of changes to the electronic structure (Figs. 2 and 3) [37]. It has been reported, and should be emphasized here, that any data manipulation such as background subtraction and smoothing can affect first moment magnitudes, leading to a risk of misinterpretation of small spectral changes [19]. To avoid uncertainties due to these processing methods, we provide the first-ever analysis of primary photosystem II emission data. These data sets are subject to no manipulation beyond the extraction of spectra via energy calibration of the detector. The statistical significance of the observed spectral changes is then determined using

TABLE II. Approximate number of x-ray pulses per state, per beam time.

\begin{tabular}{|c|c|c|c|c|c|c|c|}
\hline \multirow[b]{2}{*}{ Majority $S$ state } & \multirow[b]{2}{*}{ Flash } & \multicolumn{6}{|c|}{ Data sets } \\
\hline & & 1 & 2 & \multicolumn{2}{|c|}{$3^{\mathrm{a}}$} & 4 & $5^{\mathrm{b}}$ \\
\hline$\overline{S_{0}}$ & $3 F_{40 \mathrm{~ms}}$ & 43250 & 60000 & \multicolumn{2}{|c|}{55333} & 107222 & 97800 \\
\hline$S_{1}$ & 0 & 62267 & 72800 & \multicolumn{2}{|c|}{60233} & $\mathrm{n} / \mathrm{a}$ & 100000 \\
\hline$S_{2}$ & 1 & 42083 & 61556 & \multicolumn{2}{|c|}{55333} & $\mathrm{n} / \mathrm{a}$ & 88900 \\
\hline$S_{3}$ & 2 & $\mathrm{n} / \mathrm{a}$ & 62000 & 57178 & 48889 & 107222 & 95600 \\
\hline$S_{4 a}$ & $3 F_{50 \mu \mathrm{s}}$ & $\mathrm{n} / \mathrm{a}$ & $\mathrm{n} / \mathrm{a}$ & 57178 & 48889 & 111667 & 93300 \\
\hline$S_{4 b}$ & $3 F_{200 \mu \mathrm{s}}$ & $\mathrm{n} / \mathrm{a}$ & 60889 & 57178 & 48889 & 110556 & 86700 \\
\hline$S_{4}^{\prime}$ & $3 F_{500 \mu \mathrm{s}}$ & 43750 & 60889 & \multicolumn{2}{|c|}{57400} & $\mathrm{n} / \mathrm{a}$ & 91100 \\
\hline Total & & 191350 & 322334 & \multicolumn{2}{|c|}{546500} & 436667 & 653400 \\
\hline
\end{tabular}

\footnotetext{
${ }^{a}$ Additional statistics are collected for $S_{3}, S_{4 a}$, and $S_{4 b}$ for data set 3 . The columns are split to reflect the additional x-ray pulses for these states.

${ }^{\mathrm{b}}$ This data set is collected after 2015 upgrades to the optics at the BioCARS beam line which made impossible the use of the old experimental conditions and required twice shorter ( $1 / 2$ intensity) pulses to minimize the heat load on new optics components.
} 


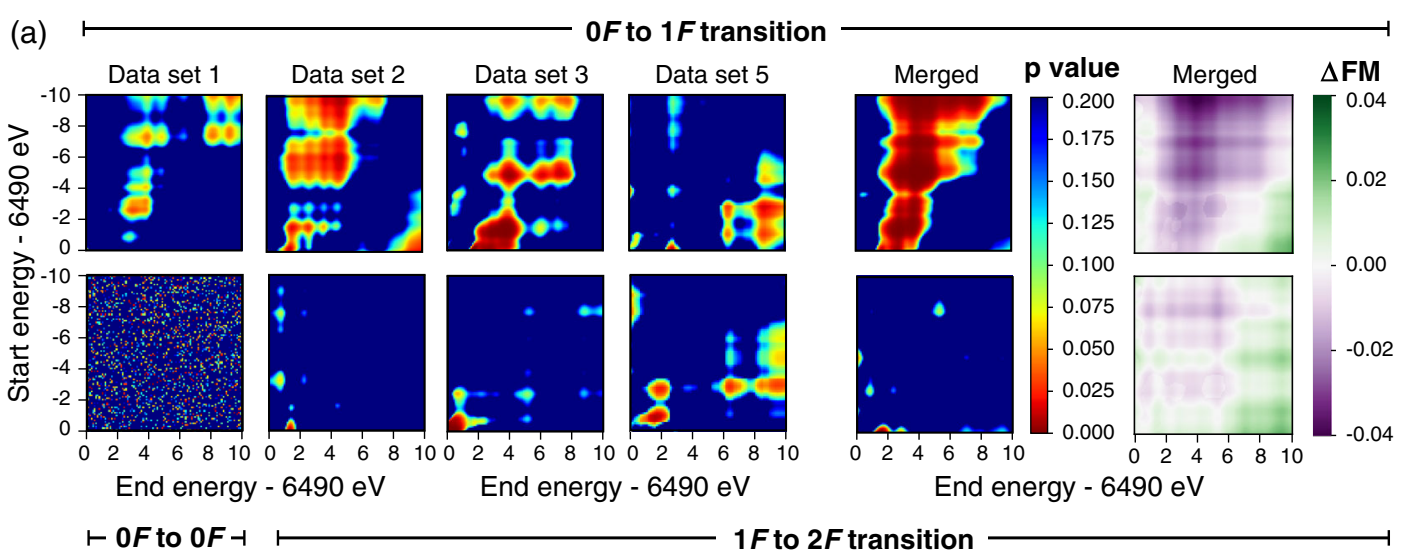

(b)
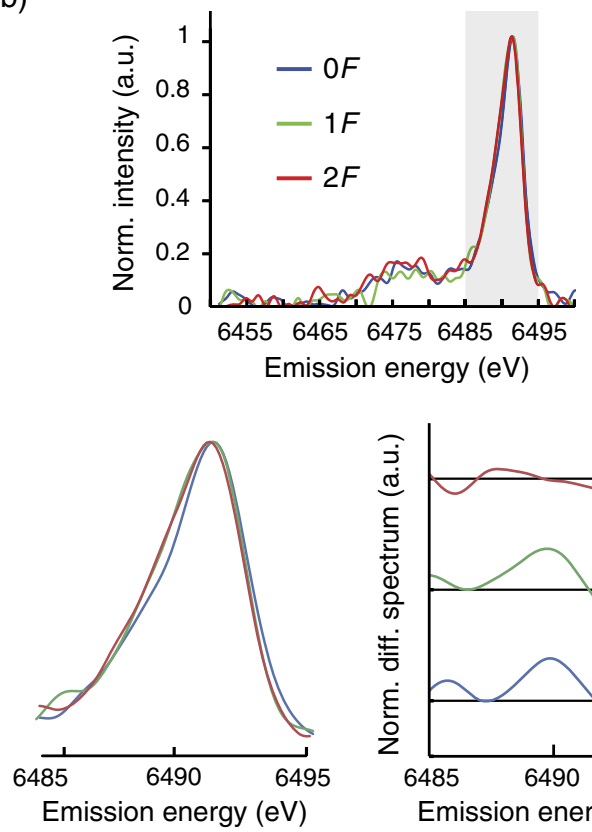

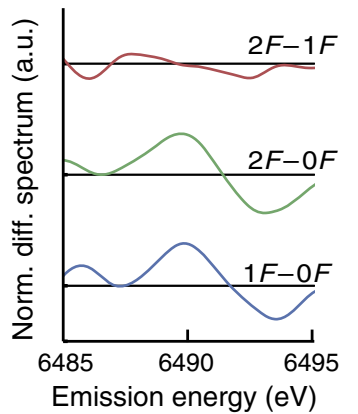

(c)
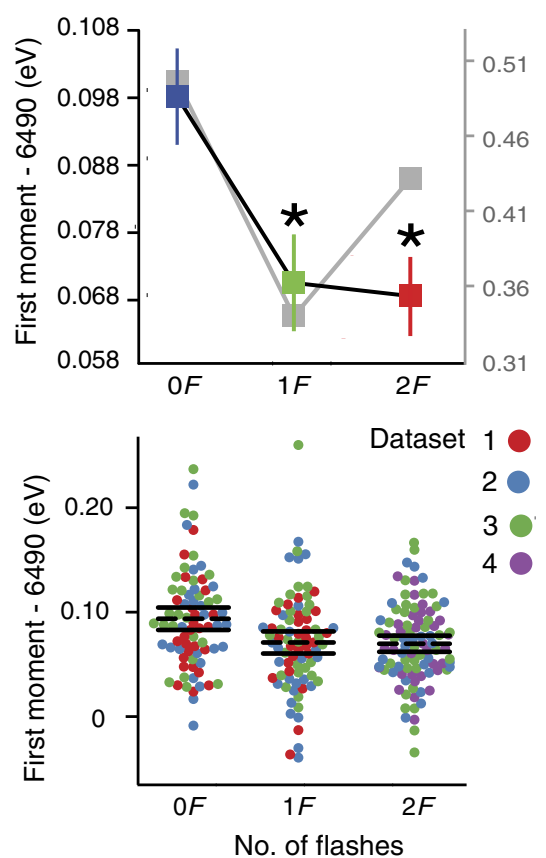

FIG. 2. Analysis of the statistical significance for $S_{1} \rightarrow S_{2} \rightarrow S_{3}$ transitions serves as a proof of concept. (a) Spectral shifts occurring as a result of $0 F \rightarrow 1 F$ and $1 F \rightarrow 2 F$ transitions are characterized using $2 \mathrm{D}$ heat maps, illustrating the $p$ value (see Table S2 for $n$ [5]) for changes to first moments calculated over the ranges defined by the $x$ and $y$ axes, i.e., start and end energy, respectively. Contours appear in plots comparing $0 F \rightarrow 1 F$ data indicate a statistically significant difference. By contrast, limited low $p$-value regions are observed for the $1 F \rightarrow 2 F$ transition, suggesting a smaller change. The directionality and magnitude of spectral shifts are shown in the final column. These $2 \mathrm{D}$ heat maps, which graphically illustrate the change in first moments $\left(\Delta \mathrm{FM}=\mathrm{FM}_{\text {postflash }}-\mathrm{FM}_{\text {preflash }}\right)$ calculated over the ranges defined by the $x$ and $y$ axes. $O F \rightarrow 1 F$ and $1 F \rightarrow 2 F$ transitions are dominated by negative, or oxidative, shifts. An example of statistical noise is presented by randomly dividing a $0 F$ data set and performing the same analysis, e.g., $0 F \rightarrow 0 F$. Relevant data from data sets 1-4 are merged to generate the final columns. Note that $0 F$ and $1 F$ data are not collected for data set 4 , while data set 5 is collected independently following a beam-line upgrade and is therefore analyzed separately; see Fig. S4 [5]. (b) Wavelet-transform smoothed and background-subtracted emission spectra for merged $0 F, 1 F$, and $2 F$ data. The region $(6.485-6.495 \mathrm{keV})$, over which the first moment is calculated, is highlighted, and a magnified inset as well as difference spectra are presented. Difference spectra are smoothed with a rolling average calculated over 14 points (approximately $\pm 0.7 \mathrm{eV}$ ). (c) (Top) Average first moments from unprocessed (color) and processed (gray) spectra. Errors are presented as SEM with $n$ given in Table III. Those moments with a statistically significant difference $(p<0.05)$ from $0 F$ data are indicated with an asterisk. (Bottom) Dot plot of first moments from raw data. Each dot represents a calculated first moment from a thread collected during the beam time corresponding to its color in the legend. Dashed lines represent the average first moment, while solid bars are the $95 \%$ confidence interval.

one-way analysis of variance (ANOVA), a simple statistical method employed broadly across many scientific disciplines. Resultant $p$ values represent the probability of falsely rejecting the null hypothesis, i.e., that a difference in the first moment between the two states listed is a random statistical variation. Thus, the lower the $p$ value, the stronger the evidence for changes in the spectra between compared data sets. In this study, we take the 


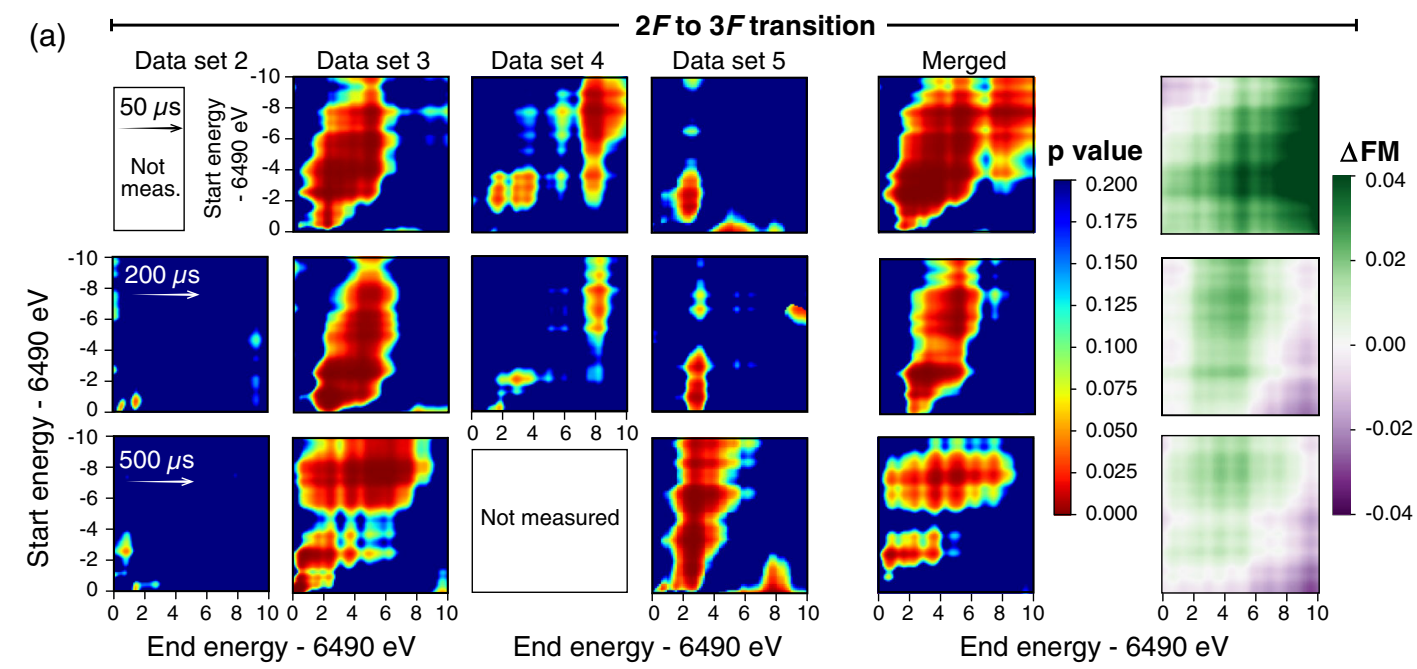

(b)
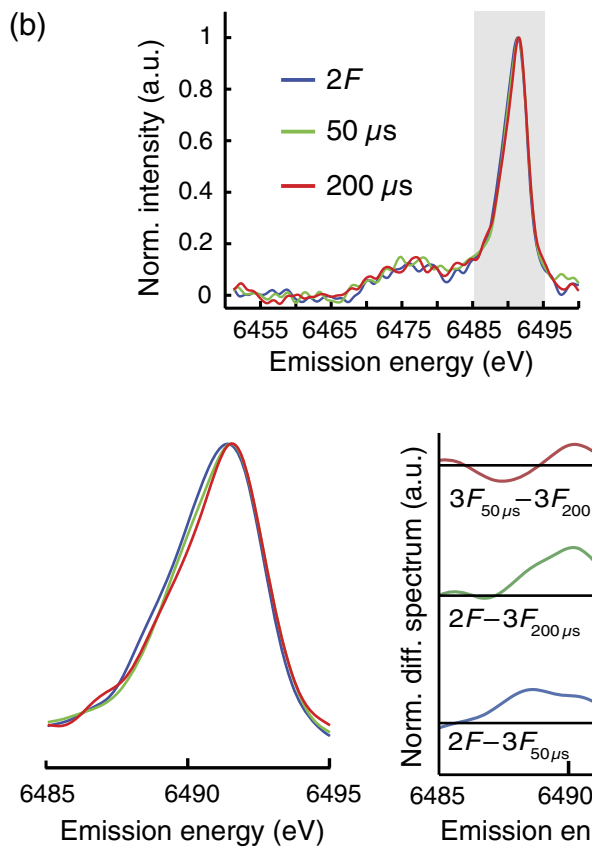

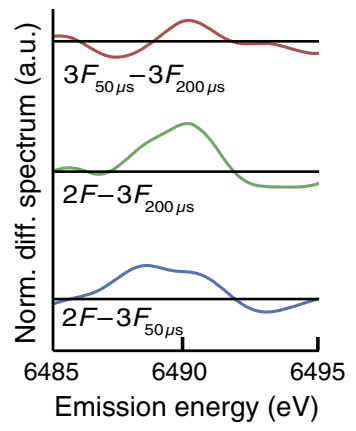

(c)
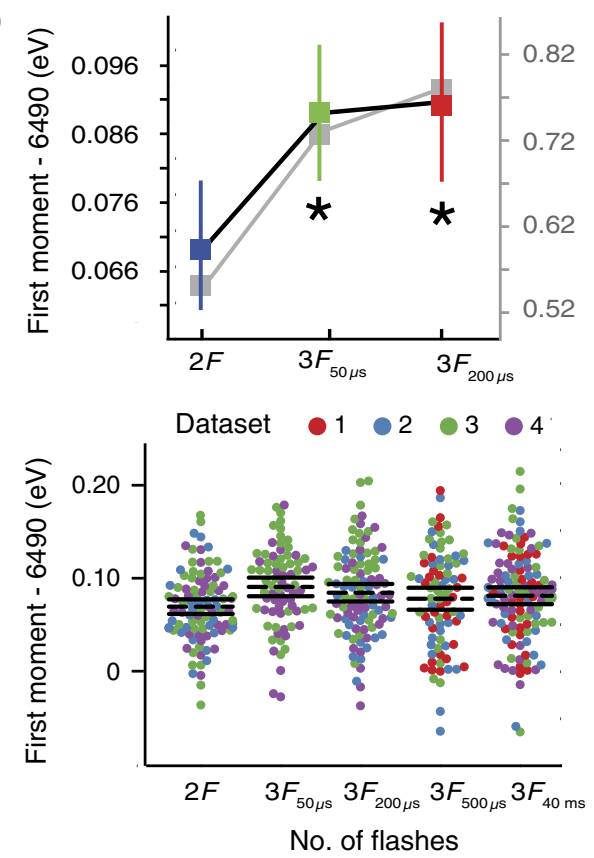

FIG. 3. Rapid onset of spectral changes observed during the $S_{3}$-to- $S_{0}$ transition. (a) Spectral shifts occurring as a result of the $2 F \rightarrow 3 F$ transition are characterized using 2D heat maps colored by $p$ value (see Table S2 [5] for $n$ ), for changes to first moments calculated over the ranges defined by the $x$ and $y$ axes, i.e., start and end energy, respectively. Strong contours are consistently observed, indicative of a statistically significant spectral change. The directionality and magnitude of spectral shifts occurring with these transitions are also shown as $2 \mathrm{D}$ heat maps, which graphically illustrate the change in first moments $\left(\Delta \mathrm{FM}=\mathrm{FM}_{\text {postflash }}-\mathrm{FM}_{\text {preflash }}\right)$ calculated over the ranges defined by the $x$ and $y$ axes. All $2 F \rightarrow 3 F$ comparisons yield primarily positive, or reductive, shifts. Determined $p$ values of approximately 0.02 (Table III) for the $200 \mu \mathrm{s}$ time point are further supported by the above heat maps; however, little information can be gleaned from these plots of data set 2, likely due to much lower x-ray shot count; see Table II. Although the $500 \mu$ s time point is equally compelling, the traditional energy range (6.485-6.495 keV) selected for reporting first moments does not deliver statistical significance (Table III). (b) Wavelet transform smoothed and background-subtracted emission spectra for merged $2 F, 3 F_{50 \mu \mathrm{s}}$, and $3 F_{200 \mu \mathrm{s}}$ data, calculated as in Fig. 2 (b). (c) (Top) Average first moments from unprocessed (color) and processed (gray) spectra. Errors are presented as SEM with $n$ given in Table III. Those moments with a statistically significant difference $(p<0.05)$ from $2 F$ data are indicated with an asterisk. (Bottom) Dot plot of first moments from raw data, presented as in Fig. 2(c). See Fig. S3 [5] for 2D plots of the 40 ms (majority $S_{0}$ state) time point.

95\% confidence interval to be significant. Background subtraction and smoothing are used only to produce figures for comparison with previous XES PS II studies, which all present significantly processed data.
While it is common to analyze first moments calculated over 6485-6495 eV, this approach fails to convey the full information content of XES spectra. To show that our trends are not dependent on a particular range chosen for 
analysis of the first moment, $p$ contours over variable energy integration ranges are shown in Figs. 2, 3, and S3 [5]. It is evident that merged data are dominated by statistically significant shifts, with the exception of $1 F$ to $2 F$, in agreement with earlier work [37]. Note that corners of the contour maps, which encompass either integration ranges outside the peak position or incomplete portions of the peak, may be influenced more significantly by noise, which is especially true for contour maps of individual beam times.

$S_{1}$-to- $S_{2}$ state transition. - The obtained $S_{1}$ state spectral shape and peak position are in good agreement with previous RT PS II measurements [28]. A comparison of $0 F$ and $1 F$ first moments evaluated by one-way ANOVA shows a reproducible, statistically significant shift of the $K \beta_{1,3}$ peak to lower energies (Table III and Figs. 2 and S4 [5]). Cryogenic measurements previously reported a $-0.059 \mathrm{eV}$ shift following spectral smoothing and background subtraction procedures [37]. Analysis of three combined data sets $(1,2$, and 3$)$ following similar data processing yields a first moment shift of $-0.16 \mathrm{eV}$ for our $0 F \rightarrow 1 F$ transition (note that data set 4 is measured without the $S_{2}$ state, and 5 is measured after the beamline update and analyzed separately). The observed shift of the $K \beta_{1,3}$ peak to lower energies following a single laser flash is likewise consistent with previous cryogenic XANES $[11,38]$ and recently published room-temperature XES [19] results for the $S_{1}$-to- $S_{2}$ state transition, both of which indicate Mn-centered oxidation. X-ray free electron laser (XFEL)-based TR XES experiments struggle to reproduce this result, likely due to a combination of lower spectrometer resolution and data-processing methods [29]. Although some XFEL studies [39,40] observe spectral shifts for $\mathrm{x}$-ray measurements made following a different number of laser flashes, they have not yet provided any new mechanistic insights. Overall, we consider our results for the $0 F \rightarrow 1 F$ transition robust and in good agreement with the previous characterization of the $S_{1}$-to- $S_{2}$ transition in which one $\mathrm{Mn}$ center is oxidized from $\mathrm{Mn}^{\mathrm{III}}$ to $\mathrm{Mn}^{\mathrm{IV}}$.
$S_{2}$-to- $S_{3}$ state transition.-For the ensuing $S$-state transition, $S_{2} \rightarrow S_{3}$, previous cryogenic measurements determined that changes to the Mn $K \beta$ emission spectrum are minimal, and a small $(-0.02 \mathrm{eV})$ shift, on the order of our systematic error (0.02 eV; see Supplemental Material [5] for more details), is reported [37]. A comparison of our $1 F$ and $2 F$ first moments, analyzed with one-way ANOVA, indicates a lack of statistical significance over most energy ranges (Table III and Figs. 2 and S4 [5]). Any associated spectral differences are likely too small to reach statistical significance under our experimental conditions. In contrast to both these measurements and earlier XAS studies [11,34,35], Zaharieva et al. recently observed a shift in the room-temperature emission spectrum equivalent to that observed during the $S_{1} \rightarrow S_{2}$ transition [19]. Although the proposed $\mathrm{Mn}$ oxidation to form $\mathrm{Mn}^{\mathrm{IV}}{ }_{4}$ in the $S_{3}$ state [41-45] [Fig. 1(a)], at its most basic, suggests a comparable XES shift for $S_{1} \rightarrow S_{2}$ and $S_{2} \rightarrow S_{3}$ transitions, we now know that the OEC undergoes a major structural change during the $S_{2} \rightarrow S_{3}$ transition from both extended $x$-ray fine structure (EXAFS) [38], femtosecond (fs) x-ray crystallography [12-14], and electron paramagnetic resonance (EPR) [44]. Studies on model compounds indicate that changes to the local spin densities associated with structural rearrangements, such as changes to the ligand environment, could obscure a Mn-centered oxidative shift $[31,46,47]$. Minimal changes to the emission spectra, observed upon the $S_{2} \rightarrow S_{3}$ transition in previous studies, are attributed to ligand-centered oxidation [37]. Low $S_{1}$-to$S_{2}$ state conversion following the first laser flash could produce equivalent changes in the $S_{1}$-to- $S_{2}$ and $S_{2}$-to- $S_{3}$ transitions observed by Zaharieva, Dau, and Haumann [18]; however, the origin of such discrepancies between reports has yet to be elucidated.

$S_{0}$ forms after $\mathrm{O}_{2}$ evolution.-To probe samples enriched with $S_{0}$, XES spectra are collected after a 40 -ms delay following a third laser flash $\left(3 F_{40 \mathrm{~ms}}\right)$. The first moment of $3 F_{40 \mathrm{~ms}}$ is consistently shifted to higher

TABLE III. $\quad p$ and $F$-statistic values from ANOVA for state-to-state comparisons between the state in the row and the state in the column for data sets 1-4 combined. $p$ values are based on the first moments over the range 6.485-6.495 keV. The number of "samples" (i.e., threads) is shown in parentheses for each state. These values are broken down by beam time in Table S2 [5]. See Table II for additional comparisons between the states based on the number of x-ray pulses per state per beam time and Figs. 2(c) and 3(c) for dot plots of all first moments used for $p$-value calculations. Values for the $F$-statistic are given in parentheses. An analysis of data set 5 is shown in Fig. S4 [5].

\begin{tabular}{|c|c|c|c|c|c|c|c|c|}
\hline & & $S_{0}$ & $S_{1}$ & $S_{2}$ & $S_{3}$ & $S_{4 a}$ & $S_{4 b}$ & $S_{4}^{\prime}$ \\
\hline Majority $S$ state $(n)$ & Flash & $3 F_{40 \mathrm{~ms}}$ & $0 F$ & $1 F$ & $2 F$ & $3 F_{50 \mu \mathrm{s}}$ & $3 F_{200 \mu \mathrm{s}}$ & $3 F_{500 \mu \mathrm{s}}$ \\
\hline$S_{0}(117)$ & $3 F_{40 \mathrm{~ms}}$ & 1 & 0.08 (3.06) & $0.06(2.19)$ & $0.06(3.60)$ & $0.17(1.82)$ & $0.63(0.23)$ & $0.66(0.19)$ \\
\hline$S_{1}(78)$ & $0 F$ & & 1 & $<0.01(9.00)$ & $<0.01$ & $0.68(0.17)$ & 0.19 (1.67) & $0.05(3.80)$ \\
\hline$S_{2}(81)$ & $1 F$ & & & 1 & $0.85(0.04)$ & $<0.01(7.27)$ & $0.02(3.68)$ & $0.98(0.82)$ \\
\hline$S_{3}(96)$ & $2 F$ & & & & 1 & $<0.01(11.32)$ & $0.02(5.87)$ & $0.22(1.50)$ \\
\hline$S_{4 a}(75)$ & $3 F_{50 \mu \mathrm{s}}$ & & & & & 1 & $0.11(0.80)$ & $0.18(2.60)$ \\
\hline$S_{4 b}(98)$ & $3 F_{200 \mu \mathrm{s}}$ & & & & & & 1 & $0.39(0.73)$ \\
\hline$S_{4}^{\prime}(82)$ & $3 F_{500 \mu \mathrm{s}}$ & & & & & & & 1 \\
\hline
\end{tabular}


energies [Figs. 3(c), S3, and S4 [5]], supporting the expected reduction of Mn. Overall, the RT TR XES results are in good agreement with previous Mn $K \beta$ emission data $[19,37]$. Having validated the experimental technique, we investigate the elusive transient $S_{3}$-to- $S_{0}$ process also initiated by three laser flashes $(3 F)$.

$\mathrm{O}-\mathrm{O}$ bond formation step.-Figure 3 depicts the earliest evolution of the OEC electronic structure following three laser flashes and the associated first moment changes of Mn $K \beta_{1,3}$ measured at $\Delta t \sim 50 \mu \mathrm{s}\left(3 F_{50 \mu \mathrm{s}}\right)$ and $\Delta t \sim$ $200 \mu \mathrm{s}\left(3 F_{200 \mu \mathrm{s}}\right)$. The trend of an increasing first moment is robust and statistically significant (Table III and Figs. 3 and S4 [5]). It is important to note that this statistically significant change occurs after the $S_{2}$-to- $S_{3}$ transition, for which our analysis does not detect a statistically significant change in the spectra (Table 3 and Fig. 2). Furthermore, the observed increase in the first moment cannot be explained by mixing of $S$ states due to poor protein advancement, as this mixing would produce an oxidative (decrease in the first moment) trend or no change; see Supplemental Material [5] for details regarding laser excitation. Based on the results of our statistical analysis, there is less than a $5 \%$ chance that this trend of an increasing first moment is merely noise. It is extremely unlikely that we would repeatedly observe $p$ values less than 0.05 if we were prevented from detecting small spectral changes due to inherent limitations of our spectroscopic setup. Random reassignment of $S$-state labels, representative of data sets limited by spectrometer resolution, for example, yields $p$ values less than 0.05 in only $5 \%$ of the random assignments, which is the expected false-positive rate. We are therefore confident that these trends are not resolution limited. To further probe the robustness of the shifts, we repeat our ANOVA analysis on randomly divided halves of the data. Recovery of statistical significance after this procedure demonstrates that there is, in fact, a $(5 \%)^{2}=$ $0.25 \%$ chance that the reported effect is due to noise.

This statistically significant spectral shift suggests that during the $S_{3}$-to- $S_{0}$ transition the OEC undergoes a significant transformation at short timescales. Changes observed at 50 and $200 \mu$ s are likely due to a short-lived isoform of the $S_{3}$ state $\left(S_{3} \mathrm{OO}\right)$, in which the $\mathrm{O}-\mathrm{O}$ bond has been formed and $\mathrm{Mn}$ centers have been reduced from the $\left(\mathrm{Mn}^{\mathrm{IV}}\right)_{4}$ state [48]. The accumulation of this species is controlled by its rate of formation $\left(k_{1}\right)$ and rate of oxidation due to electron transfer to $\operatorname{Tyr}_{Z}{ }^{\bullet}\left(k_{2}\right)$, which produces a oneelectron-more oxidized $S_{4} \mathrm{OO}$ state [Fig. 1(e)]. The first moment of the $\mathrm{Mn} K \beta_{1,3}$ line is commonly correlated with the nominal spin value of the Mn center (Fig. 4). We observe that the nominal spin of $3 F_{50 \mu \mathrm{s}}$ and $3 F_{200 \mu \mathrm{s}}$ deviates from the all $\mathrm{Mn}^{\mathrm{IV}}$ assignment reported for $S_{3}$ and represented on our plot by $\mathrm{MnO}_{2}$ oxide as well as two model compounds containing a single $\mathrm{Mn}^{\mathrm{IV}}$ center. Intriguingly, the shift between $2 F$ and $3 F_{200 \mu \mathrm{s}}$ is greater than that observed between $2 F$ and $3 F_{500 \mu \mathrm{s}}$, for which

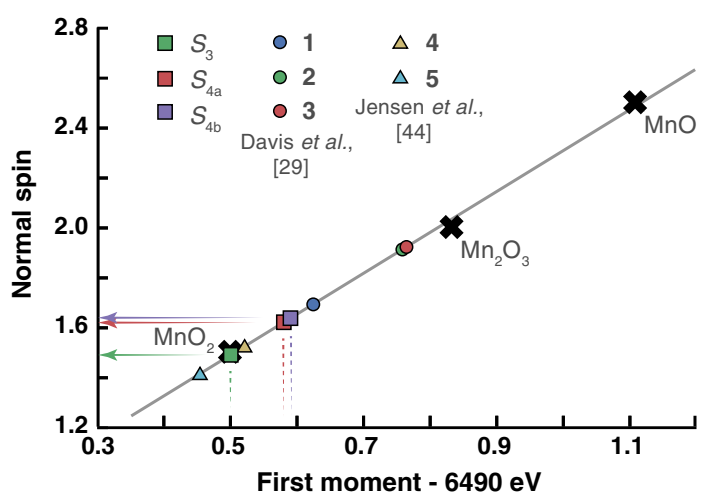

FIG. 4. Analysis of Mn $K \beta$ first moments. Placement of fully processed $2 F$ and $3 F$ data along a linear fit to a series of Mn oxide first moments empirically predicts the average spin state of $\mathrm{Mn}$ centers in the OEC. For reference, relevant Mn coordination complexes are placed along the line by using the nominal spins reported by Davis et al. and Jensen et al. $[31,46]$. Note that $S_{4 a}$ and $S_{4 b}$ correspond to $3 F$ states with $\Delta t$ between the final laser flash and an X-ray pulse of 50 and $200 \mu$ s, respectively. Compounds 1-3 are formally mixed valence $\mathrm{Mn}^{\mathrm{III}} / \mathrm{Mn}^{\mathrm{IV}}$ complexes. 1 is a di- $\mu$-oxo dimer, $\left[\mathrm{Mn}_{2} \mathrm{O}_{2} \mathrm{~L}_{4}^{\prime}\right]\left(\mathrm{ClO}_{4}\right)_{3}$, while 2 and 3 are two examples from the Mn cubane family, $\mathrm{Mn}_{4} \mathrm{O}_{4} \mathrm{~L}_{6}$. Compounds 4 and 5, by contrast, are mononuclear Mn complexes $\left[\mathrm{Mn}^{\mathrm{IV}}(\mathrm{OH})_{2}\left(\mathrm{Me}_{2} \mathrm{EBC}\right)\right]^{2+}$ and $\left[\mathrm{Mn}^{\mathrm{IV}}(\mathrm{O})(\mathrm{OH})^{-}\left(\mathrm{Me}_{2} \mathrm{EBC}\right)\right]^{+}$, respectively.

statistical significance is visible only on some plots (Fig. 3) but is not strictly demonstrated in the $6.485-6.495 \mathrm{keV}$ range. It is currently unclear whether this effect is rooted in statistical uncertainty or is simply a weak spectral change due to transient oxidation of the OEC by electron transfer to $\operatorname{Tyr}_{Z}{ }^{\cdot}\left(k_{2}\right)$ [Fig. 1(e)]. Transient oxidation would likely temporarily pause or reverse the reductive (increasing) trend in first moments.

While density functional theory (DFT) modeling has proven inconclusive regarding the formation of a $\mathrm{Mn}^{\mathrm{V}}=\mathrm{O}$ species $[24,26,49,50]$, we are unable to observe oxidation of the OEC, which would likely be associated with lower values of the first moment, at any observation time point following the third flash and measured over multiple beam times. This lack of evidence for oxidation past $2 F$ (majority state $S_{3}$ ) is consistently observed (Figs. 3, S3, and S4 [5]) thereby excluding formation of a $\mathrm{MnV}^{\mathrm{V}} \mathrm{Mn}^{\mathrm{IV}}{ }_{3}$ state kinetic intermediate. Current DFT models [23-26] also do not resolve the previously identified kinetic challenge [22]. UV-vis difference spectra show that $\operatorname{Tyr}_{Z}$ is reduced quite slowly, approximately $1 \mathrm{~ms}$ after the third flash. Given that this time constant is comparable to the rate of $\mathrm{O}_{2}$ evolution, only a very short approximately $50 \mu$ s time window remains for all bondformation dynamics and product and substrate exchange to occur.

The TR XES results detailed above cannot be explained by most DFT mechanistic models. Those which propose a $\mathrm{Mn}^{\mathrm{IV}}{ }_{4}-\mathrm{O}^{\circ}$ (oxyl radical) in place of $\mathrm{Mn}^{\mathrm{V}}=\mathrm{O}$, for 
example, predict significant activation barriers for $\mathrm{O}-\mathrm{O}$ bond formation [23,27], in disagreement with spectroscopic results that show early-onset reduction in both XES and XAS. Our results are, however, in agreement with the only other published TR studies probing the electronic structure evolution of the Mn centers via X-ray absorption spectroscopy (XAS) $[3,4,18]$. TR XAS detects no oxidation and, instead, suggests the gradual (milliseconds) reduction of the OEC, initiated $250 \mu$ s into the $S_{3}$-to$S_{0}$ transition. In contrast to the XAS study, where only two energy points along the $\mathrm{Mn} K$ edge are analyzed, we collect full spectra with high multiplicity, representing the complete electronic structure of the OEC at $\Delta t \sim 50 \mu \mathrm{s}$ and $\Delta t$ $\sim 200 \mu$ s. In addition, XAS and XES probe different electronic transitions. It is therefore possible that some early spectral changes between $t=0-200 \mu$ s may have previously escaped detection or are less pronounced in XAS due to the transient nature of the early intermediate. A more recent XES study performed at the Linac Coherent Light Source indicates no changes to the Mn $K \beta$ spectra $250 \mu$ s after the third laser excitation [51]. We attribute this discrepancy to differences in experimental conditions that will likely be clarified in the future.

To overcome the kinetic challenge, we propose that the $\mathrm{O}-\mathrm{O}$ formation step takes place in the $S_{3}$-to- $S_{0}$ transition prior to the reduction of $\operatorname{Tyr}_{Z}{ }^{*}$ [Fig. 1(e)] [30,31]. Rapid evolution of the OEC during the $S_{3}$-to- $S_{0}$ transition has long been a primary target of PS II research. Based on UVvis difference spectroscopy $[17,52]$ and TR infrared spectroscopy [16], a deprotonation event is proposed to occur early $(0-300 \mu \mathrm{s})$ during this transition. Our results do not explicitly exclude a deprotonation event but necessitate significant changes to the electronic structure of the $3 d \mathrm{Mn}$ frontier orbitals to explain the observed spectroscopic effect. The results presented here can be better rationalized if the formation of the $\left(\operatorname{Tyr}_{Z}{ }^{\circ}\right) S_{3}$ state, occurring on the order of $100 \mathrm{~ns}$ [16], triggers a sequence of events resulting in significant redox or structural changes to the OEC, such as the formation of the $\mathrm{O}-\mathrm{O}$ bond. The most recent isotope exchange studies show that substrate exchange stops early $(0-300 \mu \mathrm{s})$ in the transition [21], hinting at such a possibility. The highest activation energies are also noted in this early time window [53]. Likewise, the only molecularly defined system for water oxidation functioning with a comparative reaction rate $\left[\mathrm{Ru}(\mathrm{bda})(\mathrm{isoq})_{2}\right]$ is hypothesized to work via a radical coupling mechanism, which results in a peroxo intermediate. During the final catalytic step of this artificial system, the peroxo intermediate is further oxidized, and release of $\mathrm{O}_{2}$ follows [54]. These results suggest that the same catalytic mechanism engenders rapid $\mathrm{O}_{2}$ evolution in both biological and biomimetic systems, while at the same time preventing peroxide release due to the transient presence of the peroxo isoform.

In summary, to analyze the evolution of the photosystem II electronic structure, we observed intermediate states of photosynthetic $\mathrm{O}_{2}$ production via microsecond resolution time-resolved x-ray emission spectroscopy at a synchrotron source. Consistent with the obtained full spectra is a mechanism involving $\mathrm{O}-\mathrm{O}$ bond formation in the $S_{3}$-to$S_{0}$ transition prior to $\mathrm{Tyr}_{Z} \cdot$ reduction. This mechanism resolves the previously highlighted kinetic problems. Parallel advancements in the development of molecular catalysts for artificial photosynthesis strongly support $\mathrm{O}-\mathrm{O}$ bond formation prior to the final oxidation step of such peroxo intermediates and provide further support for the mechanistic proposal detailed herein.

This research was supported by NSF, CHE-1350909 (Y.P.) and the NSF Graduate Research Fellowship under Grant No. DGE0833366 (K. D.). G. T. S. acknowledges support from the U.S. Department of Energy, Office of Science, under Grant No. DE-SC0002194. Use of the Advanced Photon Source, an Office of Science User Facility operated for the U.S. Department of Energy (DOE) Office of Science by Argonne National Laboratory, was supported by the U.S. DOE under Contract No. DE-AC02-06CH11357. Use of the BioCARS Sector 14 was also supported by the National Institutes of Health, National Institute of General Medical Sciences Grant No. 1R24GM111072. The time-resolved setup at BioCARS was funded in part through a collaboration with Philip Anfinrud (NIH/ NIDDK). We thank Professor L. Slipchenko from Purdue University for providing computational resources and helpful discussion.

[1] B. Kok, B. Forbush, and M. McGloin, Cooperation of Charges in Photosynthetic Oxygen Evolution. I. A Linear Four Step Mechanism, Photochem. Photobiol. 11, 457 (1970).

[2] T. Wydrzynski and S. Satoh, Photosystem II: The LightDriven Water:Plastoquinone Oxidoreductase, Advances in Photosynthesis and Respiration (Springer, Dordrecht, 2005).

[3] M. Haumann, P. Liebisch, C. Muller, M. Barra, M. Grabolle, and $\mathrm{H}$. Dau, Photosynthetic $\mathrm{O}_{2}$ Formation Tracked by TimeResolved X-Ray Experiments, Science 310, 1019 (2005).

[4] M. Haumann, A. Grundmeier, I. Zaharieva, and H. Dau, Photosynthetic Water Oxidation at Elevated Dioxygen Partial Pressure Monitored by Time-Resolved X-Ray Absorption Measurements, Proc. Natl. Acad. Sci. U.S.A. 105, 17384 (2008).

[5] See Supplemental Material at http://link.aps.org/ supplemental/10.1103/PhysRevX.8.041014 for expanded materials and methods.

[6] K. N. Ferreira, T. M. Iverson, K. Maghlaoui, J. Barber, and S. Iwata, Architecture of the Photosynthetic OxygenEvolving Center, Science 303, 1831 (2004).

[7] A. Zouni, H. T. Witt, J. Kern, P. Fromme, N. Krauss, W. Saenger, and P. Orth, Crystal Structure of Photosystem II from Synechococcus elongatus at $3.8 \AA$ Resolution, Nature (London) 409, 739 (2001). 
[8] A. Guskov, J. Kern, A. Gabdulkhakov, M. Broser, A. Zouni, and W. Saenger, Cyanobacterial Photosystem II at 2.9- $\AA$ Resolution and the Role of Quinones, Lipids, Channels and Chloride, Nat. Struct. Mol. Biol. 16, 334 (2009).

[9] Y. Pushkar, J. Yano, P. Glatzel, J. Messinger, A. Lewis, K. Sauer, U. Bergmann, and V. Yachandra, Structure and Orientation of the $\mathrm{Mn}_{4} \mathrm{Ca}$ Cluster in Plant Photosystem II Membranes Studied by Polarized Range-Extended X-Ray Absorption Spectroscopy, J. Biol. Chem. 282, 7198 (2007).

[10] Y. Umena, K. Kawakami, J. R. Shen, and N. Kamiya, Crystal Structure of Oxygen-Evolving Photosystem II at a Resolution of $1.9 \AA$ A, Nature (London) 473, 55 (2011).

[11] C. Glockner, J. Kern, M. Broser, A. Zouni, V. Yachandra, and J. Yano, Structural Changes of the Oxygen-Evolving Complex in Photosystem II During the Catalytic Cycle, J. Biol. Chem. 288, 22607 (2013).

[12] C. Kupitz et al., Serial Time-Resolved Crystallography of Photosystem II Using a Femtosecond X-Ray Laser, Nature (London) 513, 261 (2014).

[13] M. Suga et al., Light-Induced Structural Changes and the Site of $\mathrm{O}=\mathrm{O}$ Bond Formation in PSII Caught by XFEL, Nature (London) 543, 131 (2017).

[14] I. D. Young et al., Structure of Photosystem II and Substrate Binding at Room Temperature, Nature (London) 540, 453 (2016).

[15] K. M. Davis and Y. N. Pushkar, Structure of the Oxygen Evolving Complex of Photosystem II at Room Temperature, J. Phys. Chem. B 119, 3492 (2015).

[16] T. Noguchi, H. Suzuki, M. Tsuno, M. Sugiura, and C. Kato, Time-Resolved Infrared Detection of the Proton and Protein Dynamics during Photosynthetic Oxygen Evolution, Biochemistry 51, 3205 (2012).

[17] F. Rappaport, M. Blancharddesce, and J. Lavergne, Kinetics of Electron-Transfer and Electrochromic Change during the Redox Transitions of the Photosynthetic OxygenEvolving Complex, Biochim. Biophys. Acta-Bioenerg. 1184, 178 (1994).

[18] I. Zaharieva, H. Dau, and M. Haumann, Sequential and Coupled Proton and Electron Transfer Events in the $S 2 \rightarrow S 3$ Transition of Photosynthetic Water Oxidation Revealed by Time-Resolved X-Ray Absorption Spectroscopy, Biochemistry 55, 6996 (2016).

[19] I. Zaharieva, P. Chernev, G. Berggren, M. Anderlund, S. Styring, H. Dau, and M. Haumann, Room-Temperature Energy-Sampling K $\beta \quad X$-Ray Emission Spectroscopy of the $\mathrm{Mn}_{4} \mathrm{Ca}$ Complex of Photosynthesis Reveals Three Manganese-Centered Oxidation Steps and Suggests a Coordination Change Prior to $\mathrm{O}_{2}$ Formation, Biochemistry 55, 4197 (2016).

[20] G. T. Babcock, R. E. Blankenship, and K. Sauer, ReactionKinetics for Positive Charge Accumulation on Water Side of Chloroplast Photosystem, FEBS Lett. 61, 286 (1976).

[21] H. Nilsson, F. Rappaport, A. Boussac, and J. Messinger, Substrate-Water Exchange in Photosystem II Is Arrested before Dioxygen Formation, Nat. Commun. 5, 4305 (2014).

[22] J. Kern and G. Renger, Photosystem II: Structure and Mechanism of the Water:Plastoquinone Oxidoreductase, Photosynth. Res. 94, 183 (2007).

[23] P. E. M. Siegbahn, Water Oxidation Mechanism in Photosystem II, Including Oxidations, Proton Release Pathways,
$\mathrm{O}-\mathrm{O}$ Bond Formation and $\mathrm{O}_{2}$ Release, Biochim. Biophys. Acta 1827, 1003 (2013).

[24] T. Saito et al., Possible Mechanisms of Water Splitting Reaction Based on Proton and Electron Release Pathways Revealed for $\mathrm{CaMn}_{4} \mathrm{O}_{5}$ Cluster of PSII Refined to $1.9 \AA$ X-Ray Resolution, Int. J. Quantum Chem. 112, 253 (2012).

[25] K. Yamaguchi et al., Full Geometry Optimizations of the Mixed-Valence $\mathrm{Ca} \mathrm{Mn}_{4} \mathrm{O}_{4} \mathrm{X}\left(\mathrm{H}_{2} \mathrm{O}\right)(4)$ ( $\mathrm{X}=\mathrm{Oh}$ or O) Cluster in Oec of PS II: Degree of Symmetry Breaking of the Labile $\mathrm{Mn}-\mathrm{X}-\mathrm{Mn}$ Bond Revealed by Several Hybrid Dft Calculations, Int. J. Quantum Chem. 113, 525 (2013).

[26] E. M. Sproviero, J. A. Gascon, J. P. McEvoy, G. W. Brudvig, and V. S. Batista, Quantum Mechanics/Molecular Mechanics Study of the Catalytic Cycle of Water Splitting in Photosystem II, J. Am. Chem. Soc. 130, 3428 (2008).

[27] P. E. M. Siegbahn, O-O Bond Formation in the $\mathrm{S}_{4}$ State of the Oxygen-Evolving Complex in Photosystem II, Chem. Eur. J. 12, 9217 (2006).

[28] K. M. Davis et al., Fast Detection Allowing Analysis of Metalloprotein Electronic Structure by X-Ray Emission Spectroscopy at Room Temperature, J. Phys. Chem. Lett. 3, 1858 (2012).

[29] J. Kern et al., Simultaneous Femtosecond X-Ray Spectroscopy and Diffraction of Photosystem II at Room Temperature, Science 340, 491 (2013).

[30] K. M. Davis et al., Rapid Evolution of the Photosystem II Electronic Structure during Water Splitting, arXiv:1506 .08862 .

[31] S. C. Jensen et al., X-Ray Emission Spectroscopy of Biomimetic Mn Coordination Complexes, J. Phys. Chem. Lett. 8, 2584 (2017).

[32] X.-c. Bai, G. McMullan, and S. H. W. Scheres, How CryoEm Is Revolutionizing Structural Biology, Trends Biochem. Sci. 40, 49 (2014).

[33] P. Glatzel and U. Bergmann, High Resolution 1s Core Hole X-Ray Spectroscopy in 3d Transition Metal ComplexesElectronic and Structural Information, Coord. Chem. Rev. 249, 65 (2005).

[34] K. M. Davis, I. Kosheleva, R. W. Henning, G. T. Seidler, and Y. Pushkar, Kinetic Modeling of the X-Ray-Induced Damage to a Metalloprotein, J. Phys. Chem. B 117, 9161 (2013).

[35] B. A. Mattern, G. T. Seidler, M. Haave, J. I. Pacold, R. A. Gordon, J. Planillo, J. Quintana, and B. Rusthoven, A Plastic Miniature X-Ray Emission Spectrometer (miniXES) Based on the Cylindrical Von Hamos Geometry, Rev. Sci. Instrum. 83, 023901 (2012).

[36] J. Messinger, W. P. Schroder, and G. Renger, StructureFunction Relations in Photosystem-II-Effects of Temperature and Chaotropic Agents on the Period 4 Oscillation of Flash-Induced Oxygen Evolution, Biochemistry 32, 7658 (1993).

[37] J. Messinger et al., Absence of Mn-Centered Oxidation in the $\mathrm{S}_{2}$ to $\mathrm{S}_{3}$ Transition: Implications for the Mechanism of Photosynthetic Water Oxidation, J. Am. Chem. Soc. 123, 7804 (2001).

[38] Y. Pushkar, J. Yano, K. Sauer, A. Boussac, and V. K. Yachandra, Structural Changes in the $\mathrm{Mn}_{4} \mathrm{Ca}$ Cluster and the Mechanism of Photosynthetic Water Splitting, Proc. Natl. Acad. Sci. U.S.A. 105, 1879 (2008). 
[39] R. Alonso-Mori et al., Towards Characterization of PhotoExcited Electron Transfer and Catalysis in Natural and Artificial Systems Using Xfels, Faraday Discuss. 194, 621 (2016).

[40] F. D. Fuller et al., Drop-on-Demand Sample Delivery for Studying Biocatalysts in Action at X-Ray Free-Electron Lasers, Nat. Methods 14, 443 (2017).

[41] T.-a. Ono, T. Noguchi, Y. Inoue, M. Kusunoki, T. Matsushita, and H. Oyanagi, X-Ray Detection of the Period-Four Cycling of the Manganese Cluster in Photosynthetic Water Oxidizing Enzyme, Science 258, 1335 (1992).

[42] L. Iuzzolino, J. Dittmer, W. Dörner, W. Meyer-Klaucke, and H. Dau, X-Ray Absorption Spectroscopy on Layered Photosystem II Membrane Particles Suggests ManganeseCentered Oxidation of the Oxygen-Evolving Complex for the $S_{0}-S_{1}, S_{1}-S_{2}$, and $S_{2}-S_{3}$ Transitions of the Water Oxidation Cycle, Biochemistry 37, 17112 (1998).

[43] M. Haumann, C. Muller, P. Liebisch, L. Iuzzolino, J. Dittmer, M. Grabolle, T. Neisius, W. Meyer-Klaucke, and H. Dau, Structural and Oxidation State Changes of the Photosystem II Manganese Complex in Four Transitions of the Water Oxidation Cycle $(S-0 \rightarrow S-1, S-1 \rightarrow S-2$, $S-2 \rightarrow S-3$, and $S-3, S-4 \rightarrow S-0)$ Characterized by X-Ray Absorption Spectroscopy at $20 \mathrm{~K}$ and Room Temperature, Biochemistry 44, 1894 (2005).

[44] N. Cox, M. Retegan, F. Neese, D. A. Pantazis, A. Boussac, and W. Lubitz, Electronic Structure of the Oxygen Evolving Complex in Photosystem II Prior to O-O Bond Formation, Science 345, 804 (2014).

[45] N. Schuth, I. Zaharieva, P. Chernev, G. Berggren, M. Anderlund, S. Styring, H. Dau, and M. Haumann, $\mathrm{K} \alpha X$ Ray Emission Spectroscopy on the Photosynthetic OxygenEvolving Complex Supports Manganese Oxidation and Water Binding in the S3 State, Inorg. Chem. 57, 10424 (2018).

[46] K. M. Davis, M. C. Palenik, L. Yan, P. F. Smith, G. T. Seidler, G. C. Dismukes, and Y. N. Pushkar, X-Ray Emission Spectroscopy of Mn Coordination Complexes Towards Interpreting the Electronic Structure of the Oxygen
Evolving Complex of Photosystem II, J. Phys. Chem. C 120, 3326 (2016).

[47] G. Vanko, T. Neisius, G. Molnar, F. Renz, S. Karpati, A. Shukla, and F.M.F. de Groot, Probing the $3 d$ Spin Momentum with X-Ray Emission Spectroscopy: The Case of Molecular-Spin Transitions, J. Phys. Chem. B 110, 11647 (2006).

[48] Y. Pushkar, K. M. Davis, and M. C. Palenik, Model of the Oxygen Evolving Complex Which Is Highly Predisposed to $O-O$ Bond Formation, J. Phys. Chem. Lett. 9, 3525 (2018).

[49] S. Yamanaka et al., Structure and Reactivity of the MixedValence $\mathrm{CaMn}_{4} \mathrm{O}_{5}\left(\mathrm{H}_{2} \mathrm{O}\right)(4)$ and $\mathrm{CaMn}_{4} \mathrm{O}_{4}(\mathrm{OH})\left(\mathrm{H}_{2} \mathrm{O}\right)(4)$ Clusters at Oxygen Evolution Complex of Photosystem II. Hybrid Dft (UB3LYP and UBHandHLYP) Calculations, Int. J. Quantum Chem. 112, 321 (2012).

[50] S. Yamanaka et al., Possible Mechanisms for the $\mathrm{O}-\mathrm{O}$ Bond Formation in Oxygen Evolution Reaction at the $\mathrm{CaMn}_{4} \mathrm{O}_{5}\left(\mathrm{H}_{2} \mathrm{O}\right)(4)$ Cluster of PSII Refined to $1.9 \AA$ $X$-Ray Resolution, Chem. Phys. Lett. 511, 138 (2011).

[51] J. Kern et al., Taking Snapshots of Photosynthetic Water Oxidation Using Femtosecond X-Ray Diffraction and Spectroscopy, Nat. Commun. 5, 4371 (2014).

[52] M. Haumann, O. Bogershausen, D. Cherepanov, R. Ahlbrink, and W. Junge, Photosynthetic Oxygen Evolution: H/D Isotope Effects and the Coupling between Electron and Proton Transfer During the Redox Reactions at the Oxidizing Side of Photosystem II, Photosynth. Res. 51, 193 (1997).

[53] H. Bao and R. L. Burnap, Structural Rearrangements Preceding Dioxygen Formation by the Water Oxidation Complex of Photosystem II, Proc. Natl. Acad. Sci. U.S.A. 112, E6139 (2015).

[54] L. Duan, F. Bozoglian, S. Mandal, B. Stewart, T. Privalov, A. Llobet, and L. Sun, A Molecular Ruthenium Catalyst with Water-Oxidation Activity Comparable to That of Photosystem II, Nat. Chem. 4, 418 (2012). 